\title{
Simple Comparison of Spectral Color Reproduction Workflows
}

\author{
Jérémie Gerhardt* and Jon Yngve Hardeberg \\ Gjøvik University College, \\ 2802 Gjøvik, Norway \\ jeremie.gerhardt@first.fraunhofer.de
}

\begin{abstract}
In this article we compare two workflows for spectral color reproduction : colorant separation (CS) followed by halftoning by scalar error diffusion (SED) of its resulting multi-colorant channel image and a second workflow by spectral vector error diffusion (sVED). Identical filters are used in both SED and sVED to diffuse the error. Gamut mapping is performed as pre-processing and the reproductions are compared to the gamut mapped spectral data. The inverse spectral Yule-Nielsen modified Neugebauer (YNSN) model is used for the colorant separation. To bring the improvement of the YNSN model upon the regular Neugebauer model into the sVED halftoning the $n$ factor is introduced in the sVED halftoning. The performances of both workflows are evaluated in term of spectral and color differences but also visually with the dot distributions obtained by the two halftoning techniques. Experimental results have shown close performances for the compared workflows in term of color and spectral differences but visually cleaner and more stable dot distributions obtained by sVED.
\end{abstract}

Keywords: Spectral color reproduction, spectral gamut mapping, colorant separation, halftoning, spectral vector error diffusion.

\section{Introduction}

With a color reproduction system it is possible to acquire the color of a scene or object under a given illuminant and reproduce it. With proper calibration and characterization of the devices involved, and not considering the problems related to color gamut limitations, it is theoretically possible to reproduce a color which will be perceived identically to the original color of the scene or object. For example, a painting and its color reproduction viewed side by side will appear identical under the illuminant used for its color acquisition even if the spectral properties of the painting pigments are different from those of the print inks. This phenomenon is called metamerism. On the other hand, if we change the illumination, then most probably the reproduction will no longer be

\footnotetext{
* Jérémie Gerhardt is working since the 1st August 2008 in Fraunhofer Institute FIRST-ISY, in Berlin, Germany (http://www.first.fraunhofer.de). This work was part of his $\mathrm{PhD}$ thesis done in the Norwegian Color Research Laboratory at HiG.
} 
perceived similar to the original. This problem can be solved in a spectral color reproduction system.

Multispectral color imaging offers the great advantage of providing the full spectral color information of the scene or object surface. Color acquisition system records the color of a scene or object surface under a given illuminant, but a multispectral color acquisition system can record the spectral reflectance and allows us to simulate the color of the scene under any illuminant. In an ideal case, after acquiring a spectral image we would like to display it or print it. For that we basically have two options: either to calculate the color rendering of our spectral image for a given illuminant and to display/print it, or to reproduce the image spectrally. This is a challenging task when for example we have made the spectral acquisition of a 2 century old painting and the colorants used at that time are not available anymore or we have lost the technical knowledge to produce them.

Multi-colorant printers offer the possibility to print the same color by various colorant combinations, i.e. metameric print is possible (note that this was already possible with a $c m y k$ printer when the grey component of a $\mathrm{cmy}$ colorant combination was replaced by black ink $k$ ). This is an advantage for colorant separation [1, 2, 3] and it allows for example to select colorant combinations minimizing colorant coverage or to optimize the separation for a given illuminant. In spectral colorant separation we aim to reduce the spectral difference between a spectral target and its reproduction, i.e. we want to reduce the metamerism. This task is performed by inverting the spectral Yule-Nielsen modified Neugebauer printer model [4], [5], 6].

Once the colorant separation has been performed the resulting multi-colorant image still has to be halftoned, and this channel independently. An alternative solution for the reproduction of spectral image is to combine the colorant separation and the halftoning in a single step: halftoning by spectral vector error diffusion [7, 8, (sVED). In our experiment we introduce the Yule-Nielsen $n$ factor in the sVED halftoning technique. Identical $n$ factor value is used at the different stages of the workflows (see diagram in Figure 1).

In the following section we will compare the reproduction of spectral data by two possible workflows for a simulated six colorants printer. The first workflow (WF1) is divided in two steps: colorant separation (CS) and halftoning by colorant channels using scalar error diffusion (SED). The second workflow (WF2) will hafltone directly the spectral image by sVED. The first step involved in the reproduction process, which is common to the two compared approaches, is a gamut mapping operation: spectral gamut mapping (sGM) is performed as pre-processing. It is the reproduction of the gamut mapped spectral data which is compared.

\section{Experiment}

The spectral images we reproduce are spectral patches. They consist of spectral image of size $512 \times 512$ pixels, each patch having a single spectral reflectance 


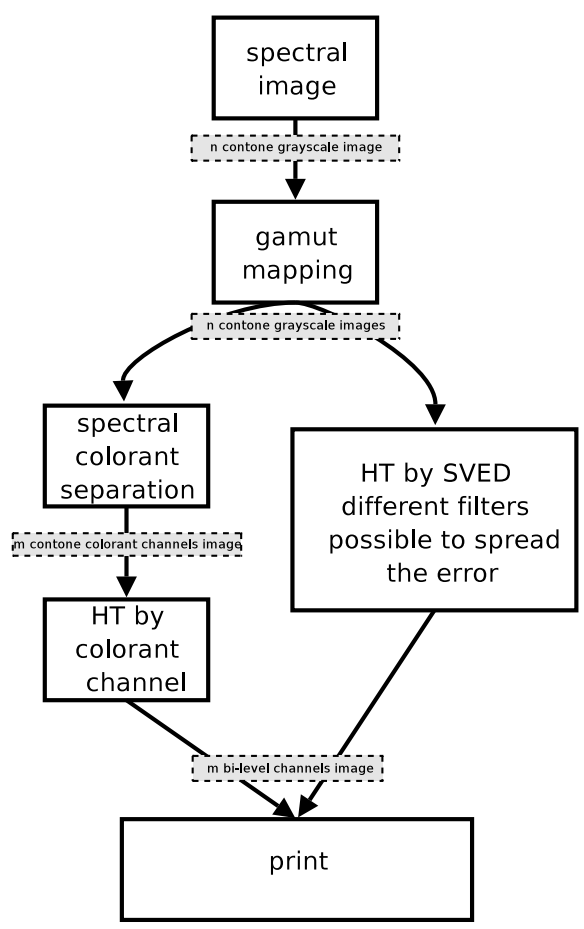

Fig. 1. Illustration of two possible workflows for the reproduction of spectral data with a $m$ colorant printer. The diagram illustrates how is transformed a spectral image into a multi bi-level colorants image.

value. The spectral reflectance targets correspond to spectral reflectance measurements extracted from a painting called La Madeleine [9].

The spectral reflectance targets have been obtained by measuring the spectral reflectances of a painting at different locations, see in Figure 2 to the left an image of the painting. Twelve samples have been selected and their spectral reproduction simulated. See in Figure 2 to the right an illustration of where the measurements were taken. The spectral reflectance corresponding to these locations are shown in Figure 4 (a). According to the workflows in Figure 1 the first step is the spectral gamut mapping. Comparison of the two workflows is based on the reproduction of the gamut mapped data.

\subsection{Spectral Gamut Mapping}

The reproduction of the spectral patches are simulated for our 6 colorants printers, see in Figure 3 the spectral reflectance the colorants. After the gamut mapping operation an original spectral reflectance $\mathbf{r}$ is replaced by its gamut mapped version $\mathbf{r}^{\prime}$ such that:

$$
\mathbf{r}^{\prime}=\mathbf{P w}^{\prime}
$$




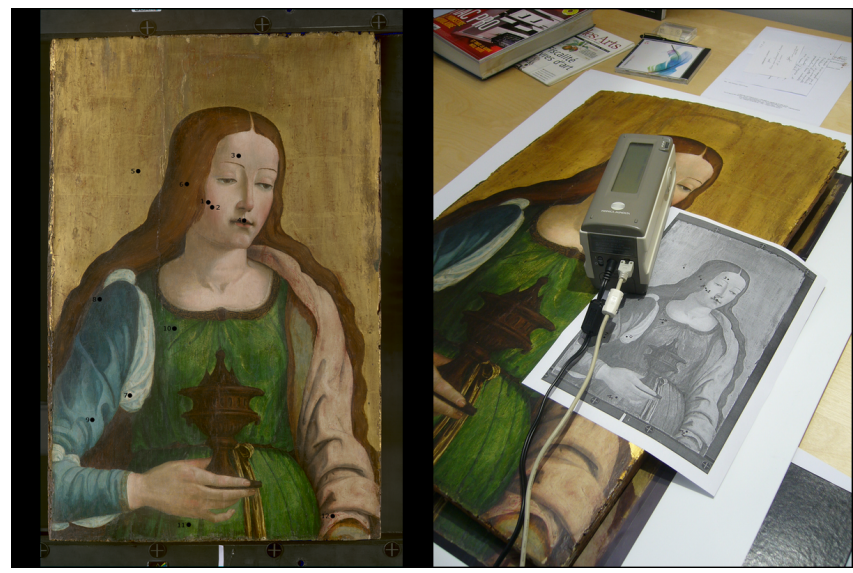

Fig. 2. Painting of La Madeleine, the 12 black spots correspond of the location where the spectral reflectances were taken

where $\mathbf{P}$ is the matrix of Neugebauer primaries (the NPs are all possible the binary combination between the available colorant of a printing system, here we have $2^{6}=64 \mathrm{NPs}$ ) and the vector of weights $\mathbf{w}^{\prime}$ is obtained while solving a convex optimization problem:

$$
\min _{\mathbf{w}^{\prime}}\left\|\mathbf{r}-\mathbf{P w}^{\prime}\right\|
$$

with the constraints on the weights $\mathbf{w}^{\prime}$ :

$$
\sum_{i=0}^{2^{m}-1} w_{i}=1 \text { and } 0 \leq w_{i} \leq 1
$$

and $m$ being the number of colorant. The $n$ factor is taking into account in the sGM operation by rising $\mathbf{r}$ and $\mathbf{P}$ to the power $1 / n$ before the optimization. In this article the $n$ factor has been set to $n=2$. As opposed to the inversion of the YNSN model by optimization we do not use the Demichel [10] equations in our gamut mapping operation [4].

The gamut mapped spectral reflectances are displayed in Figure 4 (b). Color and spectral differences between measured spectral reflectances and gamut mapped spectral reflectances are displayed in Table 1.

\subsection{WF1: Colorant Separation and Scalar Error Diffusion by Colorant Channel}

For the WF1 the colorant separation (CS) is performed for the 12 gamut mapped spectral reflectances using the linear regression iteration method (LRI) presented by [5]. From the 12 colorant combinations obtained we create 12 patches of 6 channels each and size $512 \times 512$ pixels. The final step is the halftoning operation which is performed channel independently. We use scalar error diffusion 


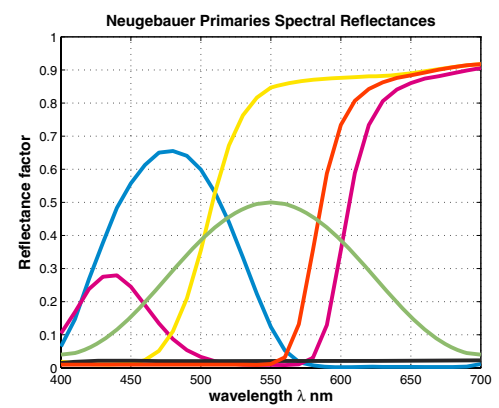

Fig. 3. Spectral reflectances of the six colorants of our simulated printing system

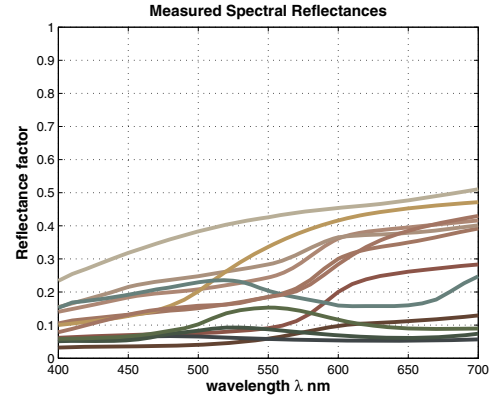

(a) Spectral reflectance measurements

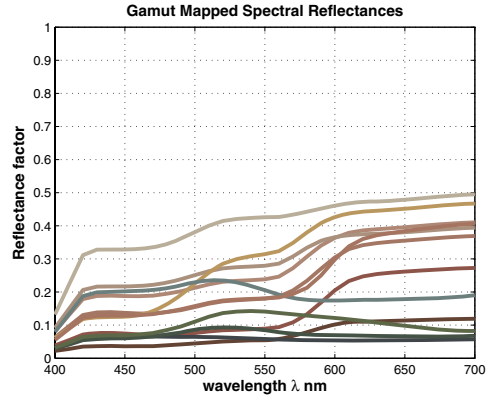

(b) Gamut mapped spectral reflectances

Fig. 4. Spectral reflectance measurements of the 12 samples in (a) and their gamut mapped version for our 6 colorants printer in (b). For each spectral reflectance displayed above the RGB color corresponds to its color rendering for illuminant D50 and CIE $19312^{\circ}$ standard observer.

(SED) halftoning technique [1] with Jarvis [12] filter to diffuse the error in the halftoning algorithm.

Each pixel of an halftoned image can be described by a multi-binary colorant combination, combination corresponding to a NP. The spectral reflectance of each patch is estimated by counting the NPs pixel's occurrences and then considering a unitary area for each patch, see the following equation:

$$
R(\lambda)=\left(\sum_{i=0}^{2^{m}-1} s_{i} P_{i}^{1 / n}(\lambda)\right)^{n}
$$

where $s_{i}$ is the area occupied by the $i t h$ Neugebauer primary $P_{i}$ and $n$ the socalled $n$ factor. Differences between the gamut mapped spectral reflectances and their simulated reproduction by CS and SED are presented in all left columns of each pair of column in Table 2 . 
Table 1. Differences between the spectral reflectance measurements and their gamut mapped version to our 6 colorants printer

\begin{tabular}{|c|c|c|c|c|c|}
\hline Samples & A & \multicolumn{3}{|c|}{$\Delta E_{a b}^{*} \quad \Delta E_{94}^{*}$} & sRMS \\
\hline 1 & 3.0 & 4.2 & 6.1 & 3.1 & 0.014 \\
\hline 2 & 3.5 & 4.9 & 6.9 & 3.5 & 0.014 \\
\hline 3 & 2.4 & 3.1 & 4.8 & 2.5 & 0.013 \\
\hline 4 & 2.9 & 4.1 & 5.7 & 2.9 & 0.009 \\
\hline 5 & 1.2 & 1.3 & 2.8 & 0.7 & 0.009 \\
\hline 6 & 2.1 & 2.9 & 3.8 & 2.0 & 0.006 \\
\hline 7 & $1.3^{3}$ & 1.4 & 0.8 & 1.2 & 0.016 \\
\hline 8 & 1.8 & 1.3 & 1.7 & 1.1 & 0.005 \\
\hline 9 & 3.5 & 2.6 & 3.3 & 1.8 & 0.023 \\
\hline 10 & 2.5 & 2.7 & 2.4 & 1.7 & 0.007 \\
\hline 11 & 4.6 & 5.7 & 5.3 & 2.8 & 0.011 \\
\hline 12 & 1. & 1.7 & 3.2 & 1.2 & 0.013 \\
\hline Av. & 2. & 3.0 & 3.9 & 2.0 & 0.012 \\
\hline Std & 1. & 1.5 & 1.9 & 0.9 & 0.005 \\
\hline Max & $4 .(1$ & 5.7 & 6.9 & 3.5 & 0.023 \\
\hline
\end{tabular}

\subsection{WF2: Spectral Vector Error Diffusion}

For this workflow we have created 12 spectral patches of size $512 \times 512$ pixels. Each spectral image have 31 channels since each spectral reflectance in our experiment is described by 31 discrete values equally spaced from $400 \mathrm{~nm}$ to 700nm.

The spectral patches are halftoned by sVED using [12 filter as in WF1 for the SED halftoning. For each pixel of a spectral image the distance to each NP is calculated, the smallest distance given colorant combination at the processed pixel. This operation is performed in a raster scan path mode, see in Figure 5] the diagram of the sVED algorithm.

Here the colorant combination selected is directly a binary combination of the 6 colorants available in our printing system, this corresponding to a command for the printer to lay down (if 1) or not (if 0 ) a drop of ink at the pixel position. Once the output is selected the difference between the processed pixel (i.e. a spectral reflectance) and the spectral reflectance of the closest NP is weighted and spread to the neighbors pixels according to the filter size.

As for the sGM operation, the CS in WF1 and while estimating the spectral reflectance of an halftoned patch we take into account the $n$ factor in the sVED algorithm: all spectral reflectances of each patch and the NPs are raised to the power $1 / n$ before to perform to halftoning.

The spectral reflectance of each patch is estimated by counting the NP pixel's occurrences and then considering a unitary area for each patch, see Equation 4. Differences between the gamut mapped spectral reflectances and their simulated reproduction by $\mathrm{sVED}$ are presented in all right columns of each pair of column in Table 2 . 


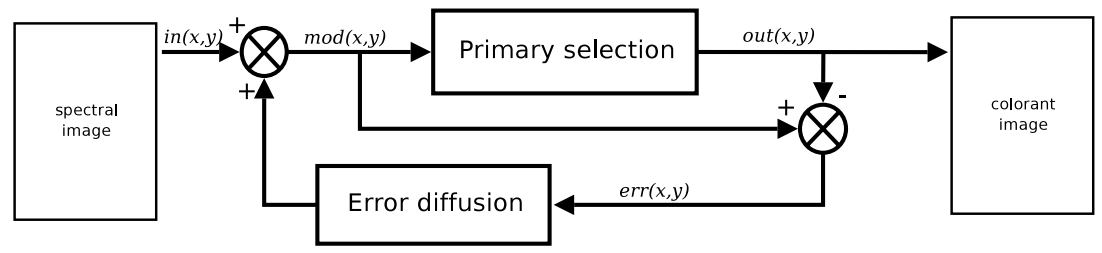

Fig. 5. The process of spectral vector error diffusion halftoning. $\operatorname{in}(x, y), \bmod (x, y)$, $\operatorname{out}(x, y)$ and $\operatorname{err}(x, y)$ are vector data representing at the position $(x, y)$ in the image the spectral reflectance of the image, the modified spectral reflectance, the spectral reflectance of the chosen primary and the spectral reflectance error.

Table 2. Differences between the gamut mapped spectral reflectances and their reproduction by CS and SED (left columns of each double column) and by sVED (right columns of each double column). The differences in bold tell us which workflow gives the smallest difference for a given sample at a given illumination condition.

\begin{tabular}{|c|c|c|c|c|c|}
\hline \multirow[b]{2}{*}{ Samples } & \multicolumn{3}{|c|}{$\Delta E_{a b}^{*}$} & \multirow{2}{*}{$\begin{array}{c}\Delta E_{94}^{*} \\
\mathrm{D} 50\end{array}$} & \multirow[t]{2}{*}{ sRMS } \\
\hline & A & D50 & FL11 & & \\
\hline 1 & $0.57 \quad \mathbf{0 . 4 6}$ & $0.51 \mathbf{0 . 3 8}$ & $\begin{array}{ll}0.43 & 0.57\end{array}$ & $0.26 \mathbf{0 . 2 5}$ & $\mathbf{0 . 0 0 2 1} 0.0036$ \\
\hline 2 & $\mathbf{0 . 3 8} \quad 0.43$ & $0.35 \mathbf{0 . 3 3}$ & $\begin{array}{ll}0.31 & 0.52\end{array}$ & $\begin{array}{ll}\mathbf{0 . 2 1} & 0.23\end{array}$ & $\begin{array}{ll}\mathbf{0 . 0 0 1 8} & 0.0039\end{array}$ \\
\hline 3 & 0.150 .41 & 0.150 .29 & 0.150 .50 & $\begin{array}{ll}0.11 & 0.23\end{array}$ & \begin{tabular}{|ll}
$\mathbf{0 . 0 0 1}$ & 0.004
\end{tabular} \\
\hline 4 & $0.68 \mathbf{0 . 5 2}$ & $0.58 \quad \mathbf{0 . 4 6}$ & 0.570 .63 & $0.35 \quad \mathbf{0 . 2 4}$ & $\begin{array}{ll}\mathbf{0 . 0 0 2 1} & 0.0028\end{array}$ \\
\hline 5 & $\mathbf{0 . 2 4} 0.40$ & $\begin{array}{lll}0.25 & 0.30\end{array}$ & 0.210 .52 & $\begin{array}{ll}\mathbf{0 . 1 6} & 0.19\end{array}$ & $\begin{array}{lll}0.0019 & 0.0041\end{array}$ \\
\hline 6 & $0.64 \mathbf{0 . 5 1}$ & $0.59 \quad \mathbf{0 . 4 4}$ & $\mathbf{0 . 5 9} 0.61$ & $0.37 \mathbf{0 . 3 0}$ & $\begin{array}{lll}0.0011 & 0.0021\end{array}$ \\
\hline 7 & $0.43 \mathbf{0 . 3 7}$ & $0.41 \quad \mathbf{0 . 2 6}$ & $\begin{array}{lll}\mathbf{0 . 3 7} & 0.48\end{array}$ & $0.31 \quad \mathbf{0 . 2 2}$ & $\begin{array}{lll}\mathbf{0 . 0 0 3 1} & 0.0043\end{array}$ \\
\hline 8 & 0.150 .44 & $\begin{array}{ll}0.19 & 0.28\end{array}$ & $\begin{array}{lll}0.16 & 0.61\end{array}$ & $\begin{array}{lll}0.18 & 0.27\end{array}$ & $\begin{array}{lll}\mathbf{0 . 0 0 0 4} & 0.0013\end{array}$ \\
\hline 9 & $0.74 \mathbf{0 . 6 0}$ & $0.96 \mathbf{0 . 5 8}$ & $0.82 \mathbf{0 . 8 0}$ & $0.81 \quad \mathbf{0 . 4 5}$ & $0.0037 \mathbf{0 . 0 0 2 7}$ \\
\hline 10 & $1.01 \quad \mathbf{0 . 7 3}$ & $1.21 \quad \mathbf{0 . 7 2}$ & $1.08 \quad \mathbf{0 . 9 3}$ & $0.85 \quad \mathbf{0 . 5 1}$ & $0.0019 \mathbf{0 . 0 0 1 5}$ \\
\hline 11 & $1.65 \quad \mathbf{0 . 6 7}$ & $1.81 \mathbf{0 . 6 5}$ & 1.810 .88 & $1.06 \mathbf{0 . 4 3}$ & $0.0038 \quad \mathbf{0 . 0 0 1 8}$ \\
\hline 12 & $\mathbf{0 . 3 1} 0.71$ & $\mathbf{0 . 3 4} 0.60$ & $\mathbf{0 . 3 6} \quad 0.99$ & $\mathbf{0 . 2 9} 0.35$ & $\mathbf{0 . 0 0 2 9} 0.0057$ \\
\hline $\mathrm{Ax}$ & $0.58 \quad 0.52$ & 0.610 .44 & $0.57 \quad 0.67$ & \begin{tabular}{|ll}
0.41 & 0.31
\end{tabular} & $0.0021 \quad 0.0032$ \\
\hline Std & $0.43 \quad 0.13$ & 0.490 .16 & $0.48 \quad 0.18$ & $\begin{array}{ll}0.31 & 0.11\end{array}$ & 0.0011 \\
\hline Max & $1.65 \quad 0.73$ & $\begin{array}{ll}1.81 & 0.72\end{array}$ & $\begin{array}{ll}1.81 & 0.99\end{array}$ & $\begin{array}{ll}1.06 & 0.51\end{array}$ & $0.0038 \quad 0.0058$ \\
\hline
\end{tabular}

\section{Results and Discussion}

The first analysis of the results, by looking at the color and spectral differences between the gamut mapped data and their simulated reproductions (see Table2) does not help to decide between WF1 or WF2. We can only observe that the average performance of the WF2 is slightly better than for the WF1 with a smaller standard deviation and a minimum maximum for all chosen illuminant.

To evaluate visually the quality of the reproduction we have created color images of the halftoned patches. Each pixel of an halftone patch (i.e. a spectral reflectance of a NP) is replaced by its RGB color rendering value for the illuminant D50 and the CIE $19312^{\circ}$ standard observer. As illustration two of the 12 patches are displayed in Figure 6 for samples 1 and 2. For all tested sample we 


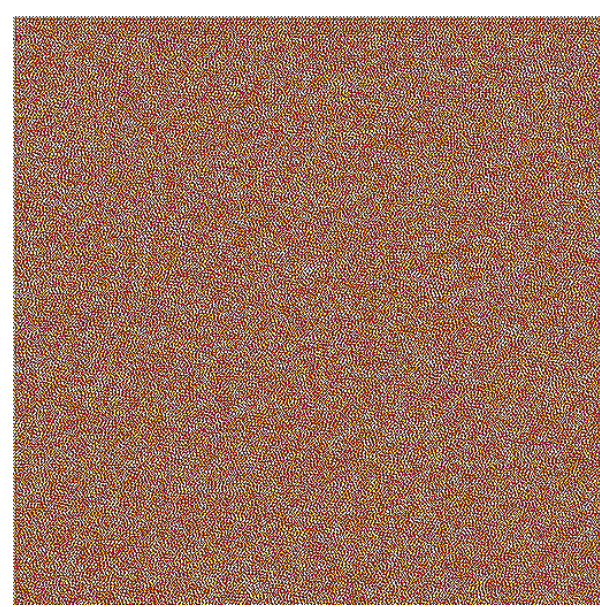

(a) HT image by SED patch 1

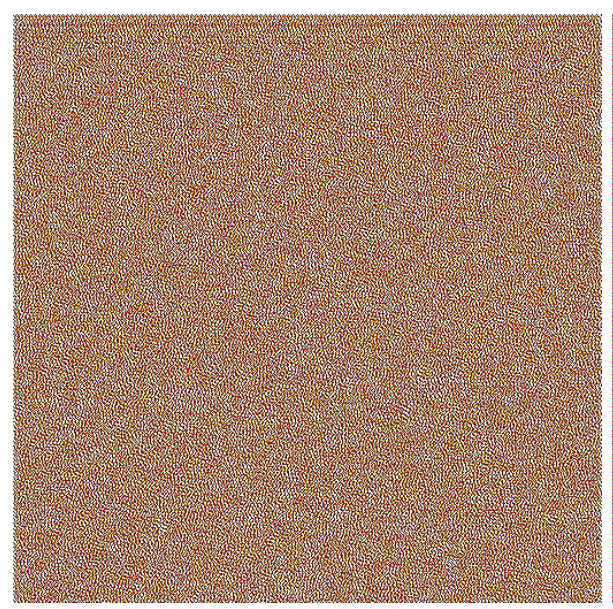

(c) HT image by SED patch 2

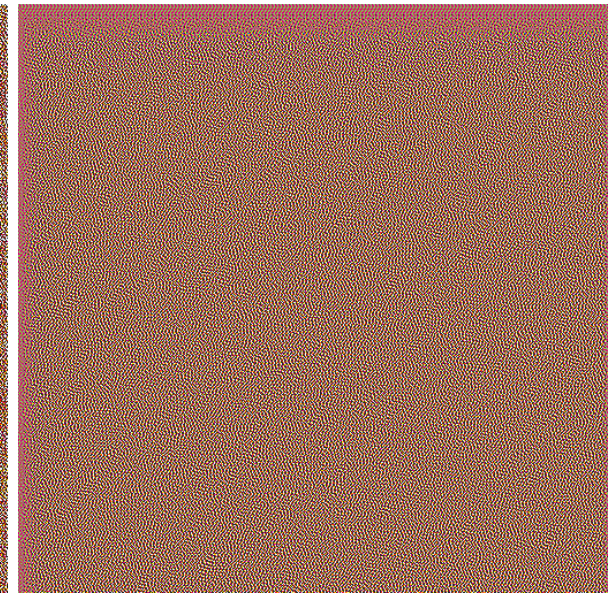

(b) HT image by SVED patch 1

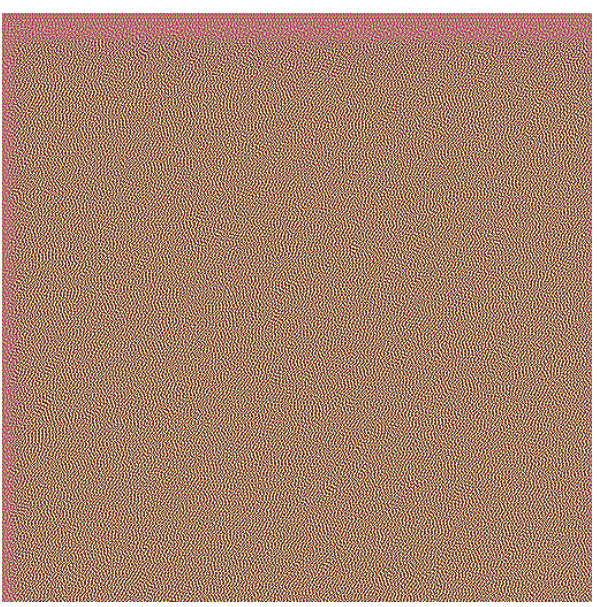

(d) HT image by SVED patch 2

Fig. 6. Color renderings of the HT images for WF1 to the left and WF2 to the right, patches 1 Figure (a) and Figure (b), patches 2 Figure (c) and Figure (d)

can observe much more pleasant spatial distributions of the NPs when halftoning by sVED has been used. The spatial NPs distribution being extremely noisy when SED halftoning was performed.

A known problem with sVED or simply VED halftoning is the slowness of error diffusion. In case of color/spectral reflectance reproduction of single patch with a single value a border effect is visible because of the path the filter is following. This border effect is also visible with SED but less stronger. The introduction of the $n$ factor before the sVED have shown a real improvement of the sVED algorithm by reaching faster a stable spatial dots distribution and a reduced border effect. 
To complete the comparison of the two proposed WFs it will be necessary to reproduce spectral images. The confrontation of sVED (including the $n$ factor) with an image will allow to compare completely the two WFs. First the sVED itself and how it behaves when the path followed the filter is crossing region of different contents (i.e. very different spectral reflectance) and how fast a stable dot distribution is reached. Secondly the computational cost and complexity of the two WFs can be evaluated and compared [13, [14].

\section{Conclusion}

The experimentation carried out in this article has allowed to compare two workflows for the reproduction of spectral images. One involving the inverse YNSN model for the colorant separation, process followed by the halftoning by SED. The second workflow has seen the use of the same parameters describing the printing system for a single operation by sVED: the NPs spectral reflectances and the $n$ factor used in the inverse printer model. Doing so the sVED halftoning and the colorant separation were performed both in $1 / n$ space. The possibility of spectral color reproduction by sVED has been already shown, but with the introduction of the $n$ factor we have have observed a clear improvement of the sVED performances in term of error visiblity by reaching faster a stable dot distribution. The slowness of error diffusion being a major drawback when vector error diffusion is the chosen halftoning technique. Further experiments have to be conducted in order to evaluate the performance on spectral images other than spectral patches.

\section{Acknowledgment}

Jérémie Gerhardt is now flying of his own wings, but he would like to thank his two directors to have selected him for his research work on spectral color reproduction and for all the helpful discussions and feedback on his work, Jon Yngve Hardeberg at HIG (Norway) and especially Francis Schmitt at ENST (France) who left us too early.

\section{References}

1. Ostromoukhov, V.: Chromaticity Gamut Enhancement by Heptatone Multi-Color Printing. In: IS\&T SPIE, pp. 139-151 (1993)

2. Agar, A.U.: Model Based Color Separation for CMYKcm Printing. In: The 9th Color Imaging Conference: Color Science and Engineering: Systems, Technologies, Applications (2001)

3. Jang, I., Son, C., Park, T., Ha, Y.: Improved Inverse Characterization of Multicolorant Printer Using Colorant Correlation. J. of Imaging Science and Technology $51,175-184(2006)$

4. Gerhardt, J., Hardeberg, J.Y.: Spectral Color Reproduction Minimizing Spectral and Perceptual Color Differences. Color Research \& Application 33, 494-504 (2008) 
5. Urban, P., Grigat, R.: Spectral-Based Color Separation Using Linear Regression Iteration. Color Research \& Application 31, 229-238 (2006)

6. Taplin, L., Berns, R.S.: Spectral Color Reproduction Based on a Six-Color Inkjet Output System. In: The Ninth Color Imaging Conference, pp. 209-212 (2001)

7. Gerhardt, J., Hardeberg, J.Y.: Spectral Colour Reproduction by Vector Error Diffusion. In: Proceedings CGIV 2006, pp. 469-473 (2006)

8. Gerhardt, J.: Reproduction spectrale de la couleur: approches par modélisation d'imprimante et par halftoning avec diffusion d'erreur vectorielle, Ecole Nationale Supérieur des Télécommunications, Paris, France (2007)

9. Dupraz, D., Ben Chouikha, M., Alquié, G.: Historic period of fine art painting detection with multispectral data and color coordinates library. In: Proceedings of Ninth International Symposium on Multispectral Colour Science and Application (2007)

10. Demichel, M.E.: Le procédé 26, 17-21 (1924)

11. Ulichney, R.: Digital Halftoning. MIT Press, Cambridge (1987)

12. Jarvis, J.F., Judice, C.N., Ninke, W.H.: A Survey of Techniques for the Display of Continuous-Tone Pictures on Bilevel Displays. Computer Graphics and Image Processing 5, 13-40 (1976)

13. Urban, P., Rosen, M.R., Berns, R.S.: Fast Spectral-Based Separation of Multispectral Images. In: IS\&T SID Fifteenth Color Imaging Conference, pp. 178-183 (2007)

14. Li, C., Luo, M.R.: Further Accelerating the Inversion of the Cellular Yule-Nielsen Modified Neugebauer Model. In: IS\&T SID Sixteenth Color Imaging Conference, pp. 277-281 (2008) 